\title{
Can lobe-specific lymph node dissection be an alternative to systematic lymph node dissection in treating early-stage non-small cell lung cancer: a comprehensive systematic review and meta-analysis?
}

\author{
Han-Yu Deng ${ }^{1,2 \#}$, Chang-Long Qin ${ }^{1 \#}$, Gang Li ${ }^{2 *}$, Guha Alai ${ }^{2}$, Yidan Lin ${ }^{2}$, Xiao-Ming Qiu ${ }^{1}$, Qinghua Zhou ${ }^{1}$ \\ ${ }^{1}$ Lung cancer center, ${ }^{2}$ Department of thoracic surgery, West China Hospital, Sichuan University, Chengdu 610041, China \\ Contributions: (I) Conception and design: YD Lin, Q Zhou; (II) Administrative support: Q Zhou; (III) Provision of study materials or patients: None; \\ (IV) Collection and assembly of data: HY Deng, CL Qin, G Li; (V) Data analysis and interpretation: G Li, G Alai, Qiu XM; (VI) Manuscript writing: \\ All authors; (VII) Final approval of manuscript: All authors. \\ \#These authors contributed equally to this work. \\ Correspondence to: Qinghua Zhou. Lung Cancer Center, West China Hospital of Sichuan University, No.37 Guoxue Alley, Chengdu 610041, China. \\ Email: zhouqh135@163.com.
}

Background: Whether lobe-specific lymph node dissection (L-SLND) could serve as an alternative to systematic lymph node dissection (SLND) in treating early-stage non-small cell lung cancer (NSCLC) remains unclear. Therefore, we conducted this comprehensive meta-analysis to compare the effect of L-SLND with that of SLND in treating early-stage NSCLC.

Methods: A systematic literature search in PubMed and Embase was conducted to identify relevant studies up to 30 November 2017. Data including 5-year overall survival (OS) and disease-free survival (DFS) rates, recurrence rates, and morbidity rate were extracted and analysed.

Results: A total of six studies [one randomized controlled trial (RCT) and five retrospective cohort studies] consisting of 2,037 patients with early-stage NSCLC were included for analysis. Meta-analysis showed that there was no significant difference of 5-year OS $[81.7 \%$ and $79.5 \%$, respectively; risk ratio $(\mathrm{RR})=1.021 ; 95 \%$ confidence interval (CI), 0.977-1.068; P=0.352] and DFS (76.4\% and 69.9\%, respectively; RR $=1.061 ; 95 \%$ CI, 0.999-1.128; $\mathrm{P}=0.054$ ) between patients treated with L-SLND and those with SLND. Moreover, there was also no significant difference of total recurrence rates $(24.3 \%$ and $25.8 \%$, respectively; RR $=0.892 ; 95 \%$ CI, 0.759-1.048; $\mathrm{P}=0.166)$ and loco-regional recurrence rates $(7.9 \%$ and $9.3 \%$, respectively; $\mathrm{RR}=0.851 ; 95 \%$ CI, 0.623-1.162; $\mathrm{P}=0.310$ ) between patients treated with L-SLND and those with SLND. However, patients treated with L-SLND yielded a significant lower morbidity rate than those treated with SLND (10.2\% and $13.5 \%$, respectively; $\mathrm{RR}=0.681 ; 95 \% \mathrm{CI},=0.521-0.888 ; \mathrm{P}=0.005)$.

Conclusions: L-SLND yielded a significantly lower risk of morbidity compared to SLND without compromising long-term oncologic outcomes based on available studies with relatively poor quality. L-SLND may serve as an alternative to SLND in treating early-stage NSCLC. Further well-conducted RCTs, however, are badly needed to confirm and update our conclusions.

Keywords: Lung cancer; early-stage; lymphadenectomy; lobe-specific; systematic; meta-analysis

Submitted Dec 20, 2017. Accepted for publication Mar 02, 2018.

doi: $10.21037 /$ jtd.2018.04.137

View this article at: http://dx.doi.org/10.21037/jtd.2018.04.137 


\section{Introduction}

Lung cancer is the leading cause of cancer related death worldwide and non-small cell lung cancer (NSCLC) is reported to account for about $85 \%$ of all lung cancers $(1,2)$. With the advancement of medical screening methods, more and more cases of early-stage NSCLC are being discovered (3). Lobectomy with systematic lymph node dissection (SLND) still remains to be the preferred treatment for patients with early-stage NSCLC (4). However, due to the evidence that the pattern of lymph node metastasis of early-stage NSCLC is lobe-specific $(5,6)$, lobe-specific lymph node dissection (L-SLND), as a more limited lymph node dissection method than SLND, was proposed for treating early-stage NSCLC (7-13). However, whether L-SLND could be an alternative to SLND remains controversial due to lacking of wellconducted randomized controlled trials (RCTs) comparing the effect of L-SLND with that of SLND in treating earlystage NSCLC. Previous studies have compared the effect of L-SLND with that of SLND and found that L-SLND could yield similar oncologic outcomes to SLND in treating early-stage NSCLC (7-10). However, their validities may be decreased by the limited sample size and observational study design (7-10). Even though a previous meta-analysis (14) compared the effect of L-SLND with that of SLND in their subgroup analysis by including three retrospective studies up to the year $2013(7,9,10)$, it also has the limitation of small sample size and other important clinical outcomes such as recurrence rate and morbidity rate were omitted for analysis in the meta-analysis. Therefore, in our study, we tried to conduct an up-to-date comprehensive meta-analysis investigating whether L-SLND could be an alternative to SLND in treating early-stage NSCLC. To our knowledge, this is the most comprehensive meta-analysis with largest sample size focusing on current topic.

\section{Methods}

\section{Search strategies}

Systematic computerized search of the PubMed and Embase for studies dated up to November 30, 2017, was performed with the following search terms: (systematic or complete) and (lobe-specific or selective) and (lymph node dissection or lymphadenectomy) and lung cancer. All reference lists from the studies selected by electronic searching were scanned to further identify relevant studies.

\section{Study selections}

The following criteria were used for study inclusion: (I) either RCTs or observational studies comparing L-SLND with SLND in treating patients with early-stage NSCLC; (II) sufficient data could be obtained for 5-year overall survival (OS), 5 -year disease-free survival (DFS), total recurrence rate and loco-regional recurrence rate, and morbidity rate; (III) the most recent or completed one was chosen if the studies were based on overlapping patients. The exclusion criteria were as follows: (I) paper without any relevant data that could be extracted for analysis; (II) paper that were not published in English; (III) case reports, abstracts, conference reports, reviews and experiments.

\section{Data extraction and quality assessment}

Two authors (HY Deng \& G Li) independently extracted data from included studies and compared the results. Discrepancies were resolved by third author (G Alai) adjudication, in order to avoid bias. Data were carefully retrieved from full articles by using a standardized data collection form. The following data were collected from each study: first author, year of publication, number of the patients, preoperative staging method, age, study design and follow-up time. The outcomes included 5-year OS, 5-year DFS, total recurrence rate and loco-regional recurrence rate, and morbidity rate. The Jadad scale (15) was used for the quality assessment of RCTs, which contained randomization ( $0-2$ points), blinding of the studies (0-2 points), and withdrawals ( $0-1$ point), and the study with high quality was defined as a study with a quality score $\geq 3$ points. The quality assessment and risk-of-bias analysis of observational studies was evaluated by The NewcastleOttawa Scale (NOS) as we previously describe (3) which consists of three factors: patient selection, comparability of the study groups, and assessment of outcome. A score of 0-9 (allocated as stars) was assigned to each study, and the high-quality study was defined as a study with quality scores $\geq 7$ (Tables 1,2). The name of the first author and the year of publication of the article were used for identification.

\section{Statistical analysis}

All analyses were performed in line with PRISMA guidelines (16) by using the STATA 12.0 package (StataCorp, College Station, TX, USA). For dichotomous data, such as 5-year OS and DFS, total recurrence rate and 
Table 1 Characteristics of the included studies in this meta-analysis

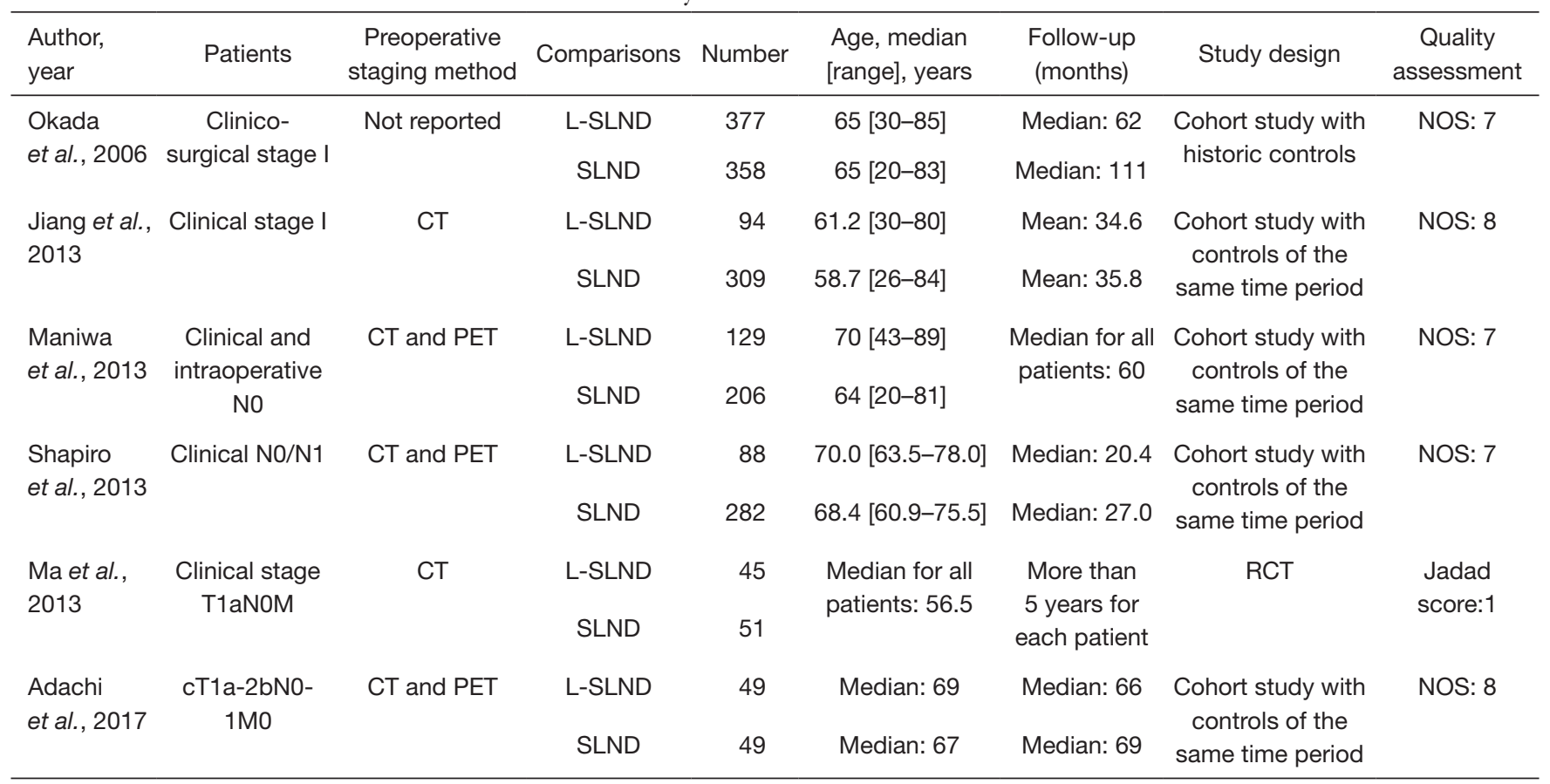

CT, computed tomography; PET, positron emission tomography; L-SLND, lobe-specific lymph node dissection; SLND, systematic lymph node dissection; NOS, Newcastle-Ottawa Scale; RCT, randomized controlled trial.

Table 2 Risk of bias assessment of the included cohort studies

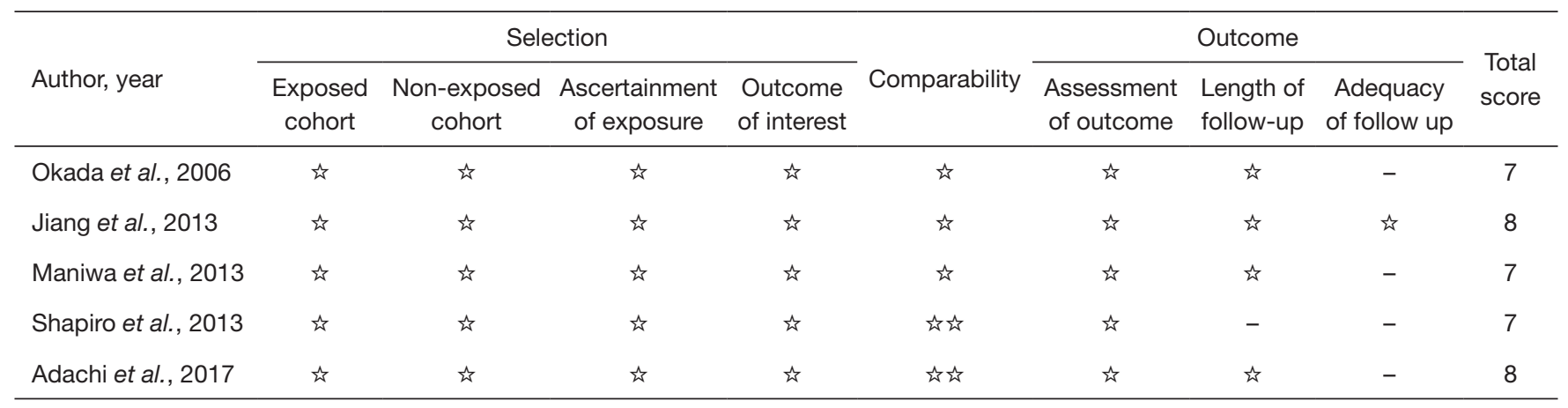

Risk of bias was evaluated with use of the Newcastle-Ottawa Scale. A score of 7 or higher indicates a low risk of bias.

loco-regional recurrence rate, and morbidity rate, risk ratio (RR) with 95\% CI were used, and 5-year OS and DFS rate were all extracted directly from the text or Kaplan-Meier curve reported in the individual studies. For each study, the between-study heterogeneity was assessed using $\chi^{2}$-based $\mathrm{Q}$ statistics and the $\mathrm{I}^{2}$ test. Random effects models were used because of the high heterogeneity of the studies $(\mathrm{P}<0.1$ or $\left.\mathrm{I}^{2}>50 \%\right)$. Otherwise, fixed effects models were used. Sensitivity analysis was performed by sequential removal of each study. A funnel plot was used to estimate potential publication bias. Asymmetry of the funnel plot was tested by Begg's test and Egger's test (17). Statistical significance was set at $\mathrm{P}<0.05$.

\section{Results}

\section{Description of studies}

A flow chart of our analysis was shown in Figure 1. A total of 132 papers were found after initial search. After initial 


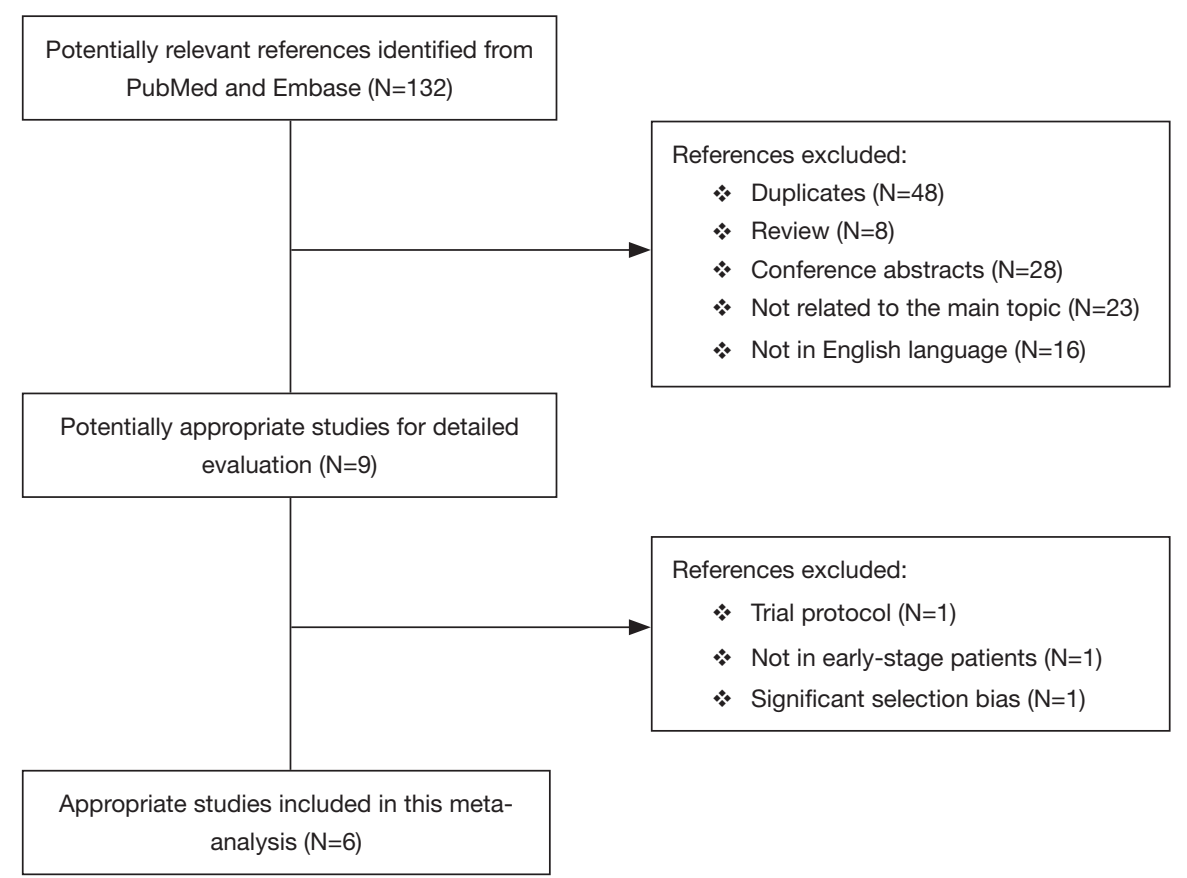

Figure 1 A flow chart showed the progress of trials through the review.

evaluation, we found nine papers for detailed evaluation. One paper (18) was excluded because it only showed a protocol for conducting an RCT comparing L-SLND and SLND, and one paper (19) was also excluded because it focused on patients with resectable localized NSCLC not specifically on patients with early-stage NSCLC. Another paper (12) was also excluded for analysis due to its significant selection bias as we would discuss it later. Finally, a total of six studies (including one RCT and five retrospective cohort studies) with a total of 2,037 patients were included for analysis $(7-11,13)$. The main characteristics extracted from these included studies were listed in Table 1 . Nearly all those studies applied computed tomography with/without positron emission tomography for preoperative staging. All patients in these studies had an early-stage NSCLC (clinical stage I or stage II), and the mean ages in both L-SLND group and SLND group were almost comparable. For SLND group, complete mediastinal lymph node dissection was performed according to the proposed definition (20), while for L-SLND group, selective mediastinal lymph node dissection was performed as follow: right upper lobar tumor (stations $2 \mathrm{R}$ and $4 \mathrm{R}$ ), left upper lobar tumor (stations 5 and 6), and right and left lower lobar tumor (stations 7, 8, and 9). All of these included studies had a relatively long follow-up time. Among those cohort studies, four used controls of the same time period for comparison while only one used historic controls. The data analysed in these studies consisted of 5-year OS and DFS, total recurrence rate and loco-regional recurrence rate, and morbidity rate (Table 3). The 5-year OS was reported by all of the included studies, while 5 -year DFS was reported in four studies. The rates of total recurrence and loco-regional recurrence, and morbidity could be obtained from five studies.

\section{Quality assessment and risk of bias}

The quality assessment and risk-of-bias analysis of the included studies were evaluated by Jadad scale for RCT and NOS for those cohort studies. The only one RCT by Ma et al. (11) mentioned randomization but did not mention withdrawal of the patients or study blinding. Therefore, it only yielded 1 score, indicating a low quality. Quality assessment of the included cohort studies was listed in Table 2. All those cohort studies $(7-10,13)$ were ranked as studies with high quality, indicating a low risk of bias.

\section{Meta-analysis of the effects of L-SLND versus SLND in treating early-stage NSCLC}

Because only one RCT with low quality was included, it 
Table 3 Main outcomes extracted from the studies included in our meta-analysis

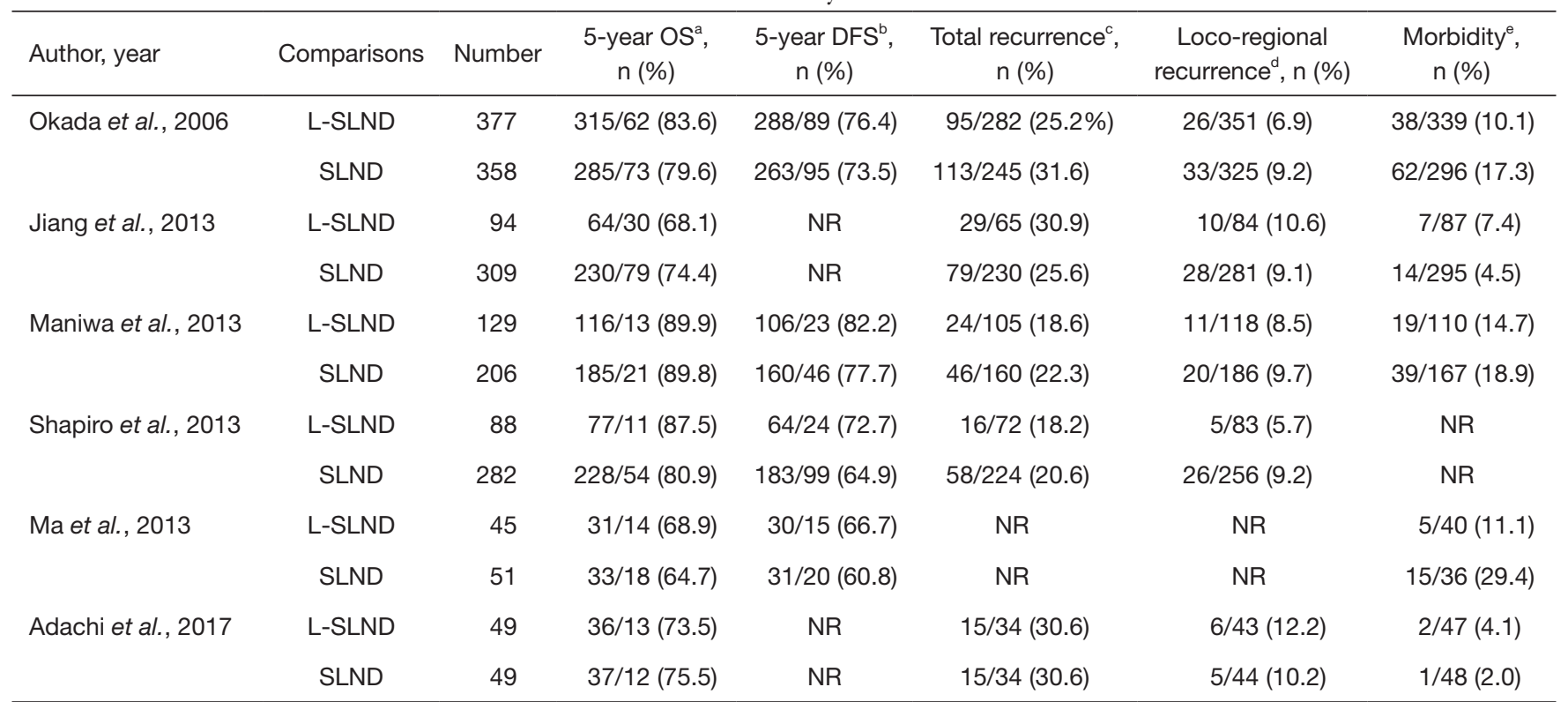

a , expressed as no. alive/no. dead and percentage; ${ }^{\text {b }}$, expressed as no. alive without disease/no. with other status and percentage;

${ }^{c}$, expressed as no. with recurrence/no. without and percentage; ${ }^{d}$, expressed as no. with loco-regional recurrence/no. without and percentage; ${ }^{e}$, expressed as no. with morbidity/no. without and percentage. OS, overall survival; DFS, disease-free survival; L-SLND, lobespecific lymph node dissection; SLND, systematic lymph node dissection; NR, not reported.

was analysed together with cohort studies in our metaanalysis. Therefore, six studies reported 5 -year OS of patients with early-stage NSCLC receiving either L-SLND or SLND with a total of 2,037 patients. Five-year OS rates for patients treated with L-SLND and SLND were 81.7\% and $79.5 \%$, respectively. There was no significant difference of 5 -year OS between patients treated with L-SLND and those with SLND (fixed effects: RR $=1.021 ; 95 \%$ CI, $0.977-$ 1.068; $\mathrm{P}=0.352 ; \mathrm{I}^{2}=0 \%$ ) (Figure $2 A$ ). Four studies reported 5 -year DFS of the patients receiving either L-SLND or SLND with a total of 1,536 patients. There was also no significant difference of 5 -year DFS between L-SLND group and SLND group (76.4\% and 69.9\%, respectively) (fixed effects: $\mathrm{RR}=1.061 ; 95 \% \mathrm{CI}, 0.999-1.128 ; \mathrm{P}=0.054$; $\mathrm{I}^{2}=0 \%$ ) (Figure $2 B$ ). Five studies reported the rates of total recurrence, loco-regional recurrence, and morbidity. There was no significant difference between patients treated with L-SLND and those with SLND in total recurrence rate (24.3\% and $25.8 \%$, respectively) (fixed effects: $\mathrm{RR}=0.892$; 95\% CI, 0.759-1.048; $\mathrm{P}=0.166 ; \mathrm{I}^{2}=0 \%$ ) (Figure $2 C$ ) and loco-regional recurrence rate $(7.9 \%$ and $9.3 \%$, respectively) (fixed effects: $\mathrm{RR}=0.851 ; 95 \% \mathrm{CI}, 0.623-1.162 ; \mathrm{P}=0.310$; $\mathrm{I}^{2}=0 \%$ ) (Figure $2 D$ ). However, patients treated with L-SLND yielded a significant lower rate of morbidity than those treated with SLND (10.2\% and $13.5 \%$, respectively) (fixed effects: $\mathrm{RR}=0.681 ; 95 \% \mathrm{CI}, 0.521-0.888 ; \mathrm{P}=0.005$; $\mathrm{I}^{2}=44.1 \%$ ) (Figure 3). No obvious heterogeneities were observed in the analysis of both oncologic and perioperative results.

\section{Sensitivity analysis and publication bias}

We conducted the sensitivity analysis by sequential removal of each study to evaluate the stability of our results based on 5-year OS and DFS, and it showed that sequential removal of each study did not change the survival outcomes of primary analysis (Figure 4). Publication bias was tested and the funnel plot for the analysis of 5-year OS had a symmetrical appearance (Begg's test: $\mathrm{P}=0.851$; Egger's test: $\mathrm{P}=0.560$ ) (Figure 5), which indicated no publication bias.

\section{Discussion}

Lobectomy with SLND still remains to be the standard treatment for early-stage NSCLC $(3,4)$. However, the optimal extent of lymph node dissection for earlystage NSCLC remains controversial as limited lymph node dissection methods (such as lymph node sampling 

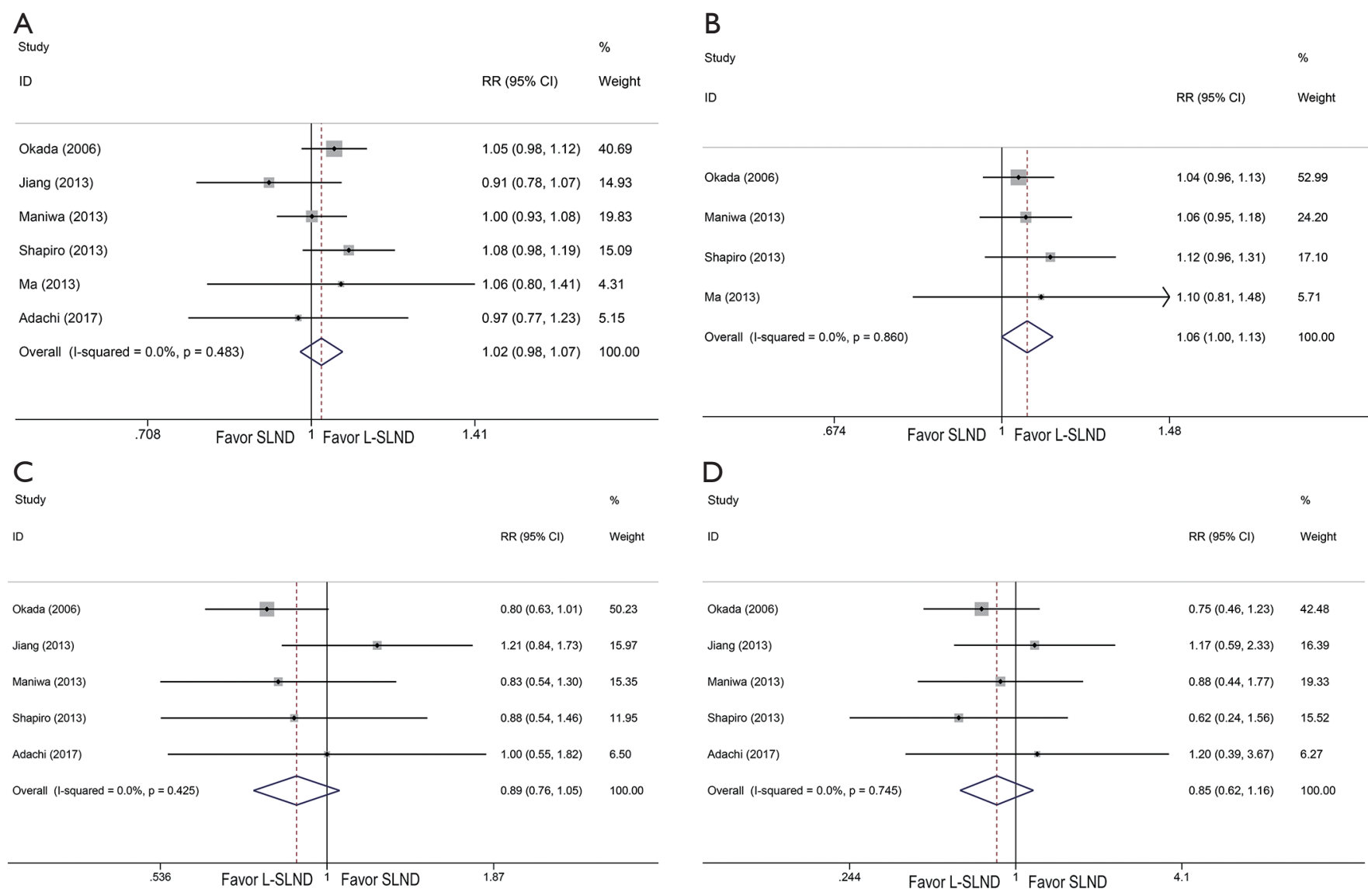

Figure 2 Forest plot of 5-year overall survival rate (A), 5-year disease-free survival rate (B), total recurrence rate (C), and loco-regional recurrence rate (D) in patients treated with lobe-specific lymph node dissection compared with systematic lymph node dissection. SLND, systematic lymph node dissection; L-SLND, lobe-specific lymph node dissection.

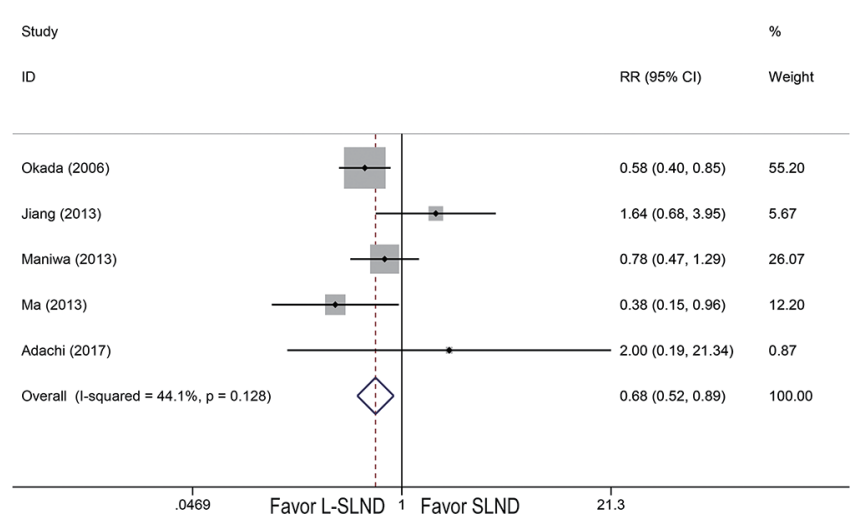

Figure 3 Forest plot of morbidity rate in patients treated with lobe-specific lymph node dissection compared systematic lymph node dissection. L-SLND, lobe-specific lymph node dissection; SLND, systematic lymph node dissection. and L-SLND) were introduced and previous studies demonstrated that SLND did not improve survival in patients with early-stage NSCLC compared to these limited lymph node dissection methods (13,21). Even though previous meta-analysis (14) tried to compare the effect of L-SLND with that of SLND in treating earlystage NSCLC by pooling available relevant studies, it only included three retrospective cohort studies $(7,9,10)$ with a limited sample size due to lacking of well-conducted RCTs. Apart from the three cohort studies $(7,9,10)$ included in previous meta-analysis, recently, more and more relevant studies comparing L-SLND with SLND have been published adding more evidence to the analysing pool $(8,12,13)$. Moreover, previous meta-analysis (14) did not compare the recurrence and perioperative outcomes 

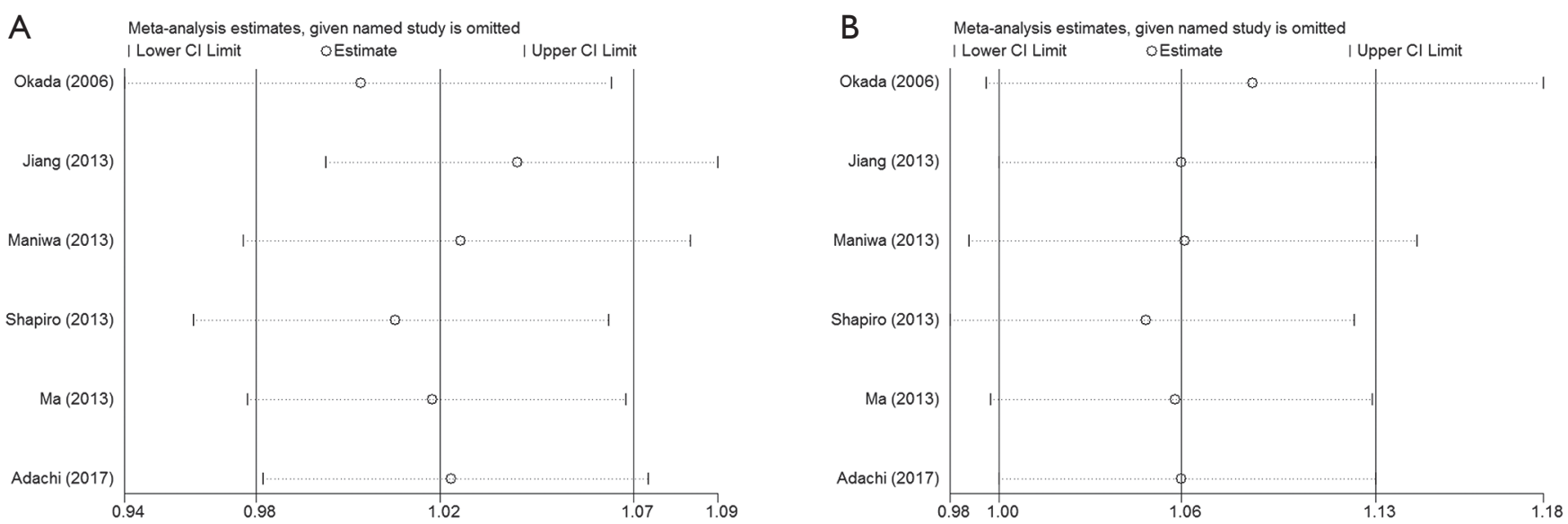

Figure 4 Sensitivity analysis for 5-year overall survival rate (A) and 5-year disease-free survival rate (B).

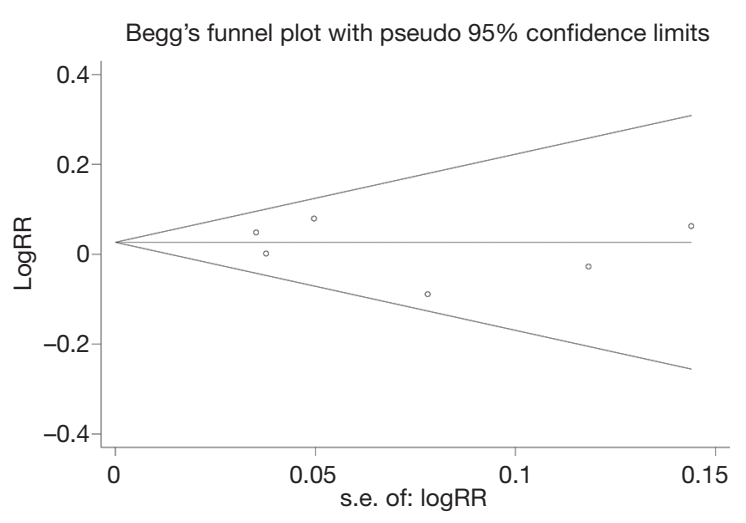

Figure 5 Funnel plot of the included studies for the analysis of 5 -year overall survival rate. Begg's test: $\mathrm{P}=0.851$; Egger's test: $\mathrm{P}=0.560$.

between L-SLND and SLND. Therefore, we conducted this updated meta-analysis by pooling the most upto date studies not only focusing on the comparison of oncologic outcomes of L-SLND and SLND but also the perioperative outcomes. To our knowledge, this is the most comprehensive meta-analysis with largest sample size focusing on current topic.

In our meta-analysis, we initially found seven studies that fitted our inclusion criteria (7-13). However, after careful evaluation, one recently published study by Hishida et al. (12) comparing the effect of L-SLND with that of SLND in Japanese patients with clinical stage I-II NSCLC from multi-institution registration with the largest sample size (a total of 5,392 patients) were excluded for final meta-analysis for the following reasons: first, one biggest limitation of this study is patient selection bias. This study was conducted from multiple institutions where the choice for patients receiving L-SLND was based not on uniform criteria but rather on the judgment at each institution. Therefore, they admitted that the favorable OS in L-SLND in their results was derived from patient selection bias. Another limitation is that the baseline characteristics between L-SLND and SLND groups were far from comparable. L-SLND group had significantly smaller tumor size and more stage IA patients than SLND groups. Finally, in the event of intraoperative suspicion of nodal involvement or if greater tumor extent was found, the planned L-SLND was converted to SLND, which would systematically bias the results in favor of L-SLND. Considering the effect of its large sample size, this cohort study was excluded from final analysis to avoid potential heterogeneities. Therefore, we finally included six studies (one RCT and five retrospective cohort studies) for analysis and because the only one RCT (11) had a low quality with limited sample size, it was analysed together with other five retrospective cohort studies in our meta-analysis.

In this meta-analysis, we found that L-SLND yielded similar 5 -year OS $(81.7 \%$ vs. $79.5 \%, \mathrm{P}=0.352)$ and $\mathrm{DFS}$ (76.4\% vs. $69.9 \%, \mathrm{P}=0.054)$ to SLND in treating earlystage NSCLC. Moreover, L-SLND also had similar total recurrence rate $(24.3 \%$ vs. $25.8 \%, \mathrm{P}=0.166)$ as well as loco-regional recurrence rate $(7.9 \%$ vs. $9.3 \%, \mathrm{P}=0.310)$ to SLND. However, L-SLND yielded a significantly lower risk of morbidity compared to SLND (10.2\% vs. $13.5 \%$, $\mathrm{P}=0.005)$. Our meta-analysis suggested that L-SLND had the advantage of decreasing the risk of postoperative 
complications over SLND without compromising longterm oncologic outcomes, adding to the evidence that L-SLND may serve as an alternative to SLND in treating early-stage NSCLC.

In the 1990s, Okada et al. (5) found that the pattern of lymph node metastasis of early-stage NSCLC was lobespecific and proposed the utility of selective mediastinal lymph node dissection based on lobe-specific lymphatic drainage as a less invasive lymph node dissection method. Later in 2006, Okada et al. (7) reported the first large series study comparing the effect of L-SLND with that of SLND in treating early-stage NSCLC and found that no significant differences of OS $(\mathrm{P}=0.060)$ or DFS $(\mathrm{P}=0.376)$ were found between patients treated with L-SLND and those with SLND. Since then, more and more similar studies comparing L-SLND and SLND were conducted and showed similar results that L-SLND yielded similar OS and DFS to SLND in treating early-stage NSCLC $(8-11,13)$. Moreover, Hishida et al. (12) even demonstrated that L-SLND was associated with a favorable survival (hazard ratio $=0.68 ; 95 \%$ CI, 0.60-0.77) compared to SLND. Even though their results may be biased due to patient selection, their study indicated that L-SLND is an alternative to SLND for selected patients with early-stage NSCLC (12). Even in patients with resectable localized NSCLC, L-SLND was also demonstrated to show no significant impact on survival compared with SLND (19). Taken our results together, we believe that in early-stage NSCLC patients L-SLND could yield equal long-term survival benefit to SLND. Moreover, our meta-analysis also demonstrated that L-SLND would not increase the recurrence rate of patients with early-stage NSCLC compared with SLND. Previously, L-SLND was also found to have significantly less blood loss and consume less operation time than SLND in treating early-stage NSCLC $(8,9,11)$. Interestingly, our meta-analysis found that L-SLND could significantly decrease the risk of morbidity in patients with early-stage NSCLC compared with SLND, which could be explained by the fact that the extent of dissection influences the frequency of complications and L-SLND could reduce the range of dissection compared to $\operatorname{SLND}(7,11)$, indicating the less invasiveness of L-SLND. Considering that L-SLND have the advantage of less invasiveness over SLND without compromising long-term oncologic benefits, we believe that L-SLND may serve as an alternative to SLND in treating early-stage NSCLC especially in the era of minimally invasive thoracic surgery.

There are still several limitations in our meta-analysis.
First, lacking of well-conducted RCTs, retrospective cohort studies might reduce the statistical power. Second, a potential heterogeneity was observed in the analysis of morbidity. Third, data of DFS, recurrence rate, and morbidity rate could not be obtained from all those included studies. Finally, potential selection biases were observed among those included retrospective cohort studies. As a result, our results should be interpreted with cautions. Therefore, even though there is one RCT registered recently (18), more well-conducted RCTs are needed to evaluate the effect of L-SLND in treating earlystage NSCLC.

\section{Conclusions}

Lacking of well-conducted RCTs, we could only include one poorly conducted RCT and five retrospective cohort studies in this updated comprehensive meta-analysis. We found that L-SLND yielded equal oncologic outcomes and significantly a lower risk of morbidity compared to SLND in patients with early-stage NSCLC. Therefore, L-SLND may serve as an alternative to SLND in treating earlystage NSCLC. Further well-conducted RCTs, however, are needed to confirm and update our conclusions.

\section{Acknowledgements}

Funding: This work was supported by Sino-Italy joint study on the key techniques of early diagnosis and individual precision treatment of lung cancer (grant No. 2016YFE0103400).

\section{Footnote}

Conflicts of Interest: The authors have no conflicts of interest to declare.

\section{References}

1. Torre LA, Bray F, Siegel RL, et al. Global cancer statistics, 2012. CA Cancer J Clin 2015;65:87-108.

2. Herbst RS, Heymach JV, Lippman SM. Lung cancer. N Engl J Med 2008;359:1367-80.

3. Deng HY, Wang YC, Ni PZ, et al. Radiotherapy, lobectomy or sublobar resection? A meta-analysis of the choices for treating stage I non-small-cell lung cancer. Eur J Cardiothorac Surg 2017;51:203-10.

4. Howington JA, Blum MG, Chang AC, et al. Treatment of 
stage I and II non-small cell lung cancer: Diagnosis and management of lung cancer, 3rd ed: American College of Chest Physicians evidence-based clinical practice guidelines. Chest 2013;143:e278S-313S.

5. Okada M, Tsubota N, Yoshimura M, et al. Proposal for reasonable mediastinal lymphadenectomy in bronchogenic carcinomas: role of subcarinal nodes in selective dissection. J Thorac Cardiovasc Surg 1998;116:949-53.

6. Asamura H, Nakayama H, Kondo H, et al. Lobe-specific extent of systematic lymph node dissection for non-small cell lung carcinomas according to a retrospective study of metastasis and prognosis. J Thorac Cardiovasc Surg 1999;117:1102-11.

7. Okada M, Sakamoto T, Yuki T, et al. Selective mediastinal lymphadenectomy for clinico-surgical stage I non-small cell lung cancer. Ann Thorac Surg 2006;81:1028-32.

8. Jiang W, Chen X, Xi J, et al. Selective mediastinal lymphadenectomy without intraoperative frozen section examinations for clinical stage I non-small-cell lung cancer: retrospective study of 403 cases. World J Surg 2013;37:392-7.

9. Maniwa T, Okumura T, Isaka M, et al. Recurrence of mediastinal node cancer after lobe-specific systematic nodal dissection for non-small-cell lung cancer. Eur J Cardiothorac Surg 2013;44:e59-64.

10. Shapiro M, Kadakia S, Lim J, et al. Lobe-specific mediastinal nodal dissection is sufficient during lobectomy by video-assisted thoracic surgery or thoracotomy for early-stage lung cancer. Chest 2013;144:1615-21.

11. Ma W, Zhang ZJ, Li Y, et al. Comparison of lobespecific mediastinal lymphadenectomy versus systematic mediastinal lymphadenectomy for clinical stage $\mathrm{T}(1) \mathrm{a} \mathrm{N}(0)$ M(0) non-small cell lung cancer. J Cancer Res Ther 2013;9 Suppl 2:S101-5.

12. Hishida T, Miyaoka E, Yokoi K, et al. Lobe-Specific Nodal Dissection for Clinical Stage I and II NSCLC: Japanese Multi-Institutional Retrospective Study Using a Propensity
Score Analysis. J Thorac Oncol 2016;11:1529-37.

13. Adachi H, Sakamaki K, Nishii T, et al. Lobe-Specific Lymph Node Dissection as a Standard Procedure in Surgery for Non-Small Cell Lung Cancer: A Propensity Score Matching Study. J Thorac Oncol 2017;12:85-93.

14. Meng D, Zhou Z, Wang Y, et al. Lymphadenectomy for clinical early-stage non-small-cell lung cancer: a systematic review and meta-analysis. Eur J Cardiothorac Surg 2016;50:597-604.

15. Jadad AR, Moore RA, Carroll D, et al. Assessing the quality of reports of randomized clinical trials: is blinding necessary? Control Clin Trials 1996;17:1-12.

16. Moher D, Liberati A, Tetzlaff J, et al. Preferred reporting items for systematic reviews and meta-analyses: the PRISMA statement. PLoS Med 2009;6:e1000097.

17. Egger M, Davey Smith G, Schneider M, et al. Bias in meta-analysis detected by a simple, graphical test. BMJ 1997;315:629-34.

18. Hishida T, Saji H, Watanabe SI, et al. A randomized Phase III trial of lobe-specific vs. systematic nodal dissection for clinical Stage I-II non-small cell lung cancer (JCOG1413). Jpn J Clin Oncol 2018;48:190-4.

19. Ishiguro F, Matsuo K, Fukui T, et al. Effect of selective lymph node dissection based on patterns of lobe-specific lymph node metastases on patient outcome in patients with resectable non-small cell lung cancer: a large-scale retrospective cohort study applying a propensity score. J Thorac Cardiovasc Surg 2010;139:1001-6.

20. Rami-Porta R, Wittekind C, Goldstraw P. Complete resection in lung cancer surgery: proposed definition. Lung Cancer 2005;49:25-33.

21. Darling GE, Allen MS, Decker PA, et al. Randomized trial of mediastinal lymph node sampling versus complete lymphadenectomy during pulmonary resection in the patient with N0 or N1 (less than hilar) non-small cell carcinoma: results of the American College of Surgery Oncology Group Z0030 Trial. J Thorac Cardiovasc Surg 2011;141:662-70.
Cite this article as: Deng HY, Qin CL, Li G, Alai G, Lin Y, Qiu XM, Zhou Q. Can lobe-specific lymph node dissection be an alternative to systematic lymph node dissection in treating early-stage non-small cell lung cancer: a comprehensive systematic review and meta-analysis? J Thorac Dis 2018;10(5):28572865. doi: $10.21037 /$ jtd.2018.04.137 